\title{
The impact of frequent injections for hematopoietic growth factor support on patients receiving chemotherapy: an observational study
}

\author{
Susan Haithcox ${ }^{1}$, Carmella R Ramnes ${ }^{2}$, Howard Lee ${ }^{3}$, John $\mathrm{Lu}^{* 4}$ and \\ Gary H Lyman ${ }^{5}$
}

Address: ${ }^{1}$ Department of Oncology Research, Northern Indiana Cancer Research Consortium, South Bend, Indiana, 46601, USA, ${ }^{2}$ Department of Hematology-Oncology, Albany Medical Center, Albany, New York, 12208, USA, ${ }^{3}$ Division of Hematology-Oncology, University of California, Los Angeles, California, 90095, USA, ${ }^{4}$ Department of Health Economics and Epidemiology, Amgen Inc, Thousand Oaks, California, 91320, USA and ${ }^{5}$ Division of Hematology-Oncology, University of Rochester, Rochester, New York, 14627, USA

Email: Susan Haithcox - shaithcox@memorialsb.org; Carmella R Ramnes - cramnes@ordwayresearch.org; Howard Lee - hl@ucla.edu; John Lu* - zjohnlu@amgen.com; Gary H Lyman - Gary_Lyman@urmc.rochester.edu

* Corresponding author

Published: 19 September 2003

BMC Nursing 2003, 2:2

This article is available from: http://www.biomedcentral.com/l472-6955/2/2

(c) 2003 Haithcox et al; licensee BioMed Central Ltd. This is an Open Access article: verbatim copying and redistribution of this article are permitted in all media for any purpose, provided this notice is preserved along with the article's original URL.
Received: 9 April 2003

Accepted: 19 September 2003

\begin{abstract}
Background: Quality of life may be affected by daily injections of supportive hematopoietic growth factor medication, which is frequently required by patients with cancer undergoing chemotherapy. The objective of the study was to identify areas where long-acting derivatives of current proteins, which require less frequent administration, may provide advantages over existing therapies that require more frequent administration.
\end{abstract}

Methods: An observational study was conducted to assess the impact of daily injections of Filgrastim (Neupogen ${ }^{\circledR}$; Amgen Inc.) on patients' quality of life. A Subject Outcome Questionnaire was administered to patients after chemotherapy on 2 consecutive cycles. Time spent for treatment and patient attitude towards injection frequency were measured. The effect on patients' daily activities, including their ability to work, was analyzed.

Results: Thirty patients completed the first, and 24 the second, administration of the Questionnaire across 3 participating sites in the United States. The average patient time commitment for each daily injection was 78 minutes. Forty-five percent of patients were moderately to extremely bothered by travel during the first chemotherapy cycle, which increased to $59 \%$ during the second cycle. Forty-four percent and $18 \%$ of patients reported having to rearrange their daily schedules and take time off from work to accommodate each injection at least some of the time, respectively. Eighty-nine percent of the patients reported a preference for a longer-acting drug that required fewer injections.

Conclusion: Results indicate that frequent injections represent a significant burden on patients' lives and that the majority would prefer longer-acting medications that require less frequent administration and potentially fewer clinic visits. 


\section{Background}

When people are diagnosed with cancer, their daily routines, and those of their caregivers, change dramatically in order to accommodate chemotherapy treatment. Given the potential outcome of the disease, patients are often willing to make significant adjustments in their lives to integrate the numerous demands of their disease treatment plans. Understandably, this new identity as a "cancer patient" may take priority over all other roles when such sacrifice is believed to be necessary to improve the probability of a successful outcome. Thus, for patients and their caregivers, frequent trips to the clinic that necessitate rearranging schedules, taking time off from work, arranging for childcare, and missing social and family activities become an unfortunate part of their daily routines. Many trips to the clinic may only be for a single injection of supportive therapy. Although injections may seem like simple and quick procedures, in reality patients frequently expend a great deal of time and effort each day to receive them. Meanwhile, the very activities that made life full and meaningful before their diagnosis have the potential to be pushed aside for several hours at a time, as patients receive their treatment. Furthermore, balancing these activities may become stressful for patients and their caregivers. It may also become increasingly more difficult for patients to carry on with normal life if they are constantly in the clinic. Therefore, for these patients, a medication that requires less frequent administration, and offers the potential of fewer clinic visits and greater flexibility, could provide a tremendous emotional and physical advantage over existing therapies.

Although the clinical value of supportive medications such as Filgrastim (Neupogen ${ }^{\circledR}$ ) and Epoetin alfa (PRO$\mathrm{CRIT}^{\circledast}$ ) for the management of chemotherapy-induced neutropenia (CIN) and anemia (CIA), respectively, is widely accepted [1-5], the challenges associated with frequent injections that patients encounter have not been adequately addressed. Several randomized, phase 3 studies have demonstrated the safety and efficacy of Filgrastim in the setting of CIN $[1,2,6,7]$. In these studies, daily Filgrastim was administered until an absolute neutrophil count $\geq 10 \times 10^{9} / \mathrm{L}$ was documented, resulting in an average of 10 to 11 injections per chemotherapy cycle. Although Epoetin alfa is administered less frequently than Filgrastim, patients may also require frequent injections, at least once weekly. These injections may further disrupt the lives of patients, in particular those who may be required to travel to clinic for their injections.

Recently, 2 novel long-acting supportive care proteins were approved by the United States (US) Food and Drug Administration (FDA) for use by patients receiving chemotherapy. Pegfilgrastim (Neulasta ${ }^{\circledR}$ ) is used to reduce the incidence of infection, as manifested by febrile neutrope- nia, in patients with nonmyeloid malignancies receiving chemotherapy, and darbepoetin alfa (Aranesp ${ }^{\circledR}$ ) is used for the treatment of CIA in patients with nonmyeloid malignancies. Due to the longer serum half-lives, both molecules require less frequent administration than prior treatments. Pegfilgrastim is administered once per chemotherapy cycle compared with daily injections of Filgrastim, whereas darbepoetin alfa is administered weekly compared with three times weekly injections of Epoetin alfa, per the product labeling for each drug. However, in the US, Epoetin alfa is commonly administered weekly in the oncology setting [5]; similarly, a less frequent schedule (once every two weeks) for darbepoetin alfa administration has been routinely adopted [8].

This study was designed to investigate the burden associated with frequent injections on patients requiring Filgrastim therapy. The results from this study can be used to identify areas in a patient's treatment where long-acting agents could provide advantages over existing therapies that require more frequent administration.

\section{Methods \\ Study population}

Oncology patients in participating ambulatory oncology clinics who were expected to receive at least 2 cycles of Filgrastim therapy were eligible for this study; those who were unable to understand spoken English at a $6^{\text {th }}$ grade level or with severe comorbidities were excluded. Patients who met these eligibility criteria were then randomly selected and contacted for their interest in participating in the study.

The study protocol was approved by the Institutional Review Board at each of the 3 investigative sites, and written informed consent was obtained from each study participant before the protocol was initiated.

\section{Study design}

This observational study was designed to assess the impact of frequent injections on the disruption of daily activities, time commitment, physical discomfort, and satisfaction with treatment in patients receiving chemotherapy who require supportive therapy. Study participants provided answers to a self-administered Subject Outcome Questionnaire (SOQ) completed $15 \pm 3$ days after chemotherapy on 2 consecutive chemotherapy cycles. The SOQ (see additional file 1: Subject outcome questionnaire) was developed to assess the burden associated with daily injections on patients receiving Filgrastim post-chemotherapy. The items included in the questionnaire were based on results of 2 focus group studies of cancer patients who received Filgrastim during their chemotherapy treatment (data not published). Although the sample sizes of these exploratory studies were small ( 9 in one and 6 in the 
Table I: Demographic characteristics of study population

\begin{tabular}{ll}
\hline Age, years & \\
$\quad$ mean (SD) & $60.8(13.9)$ \\
range & $21-80$ \\
$\geq 65(\%)$ & $45 \%$ \\
Sex, female (\%) & $70 \%$ \\
Level of education, college degree or higher & $47 \%$ \\
Insurance coverage by Medicare & $45 \%$ \\
Annual household income, >\$50,000/year & $45 \%$ \\
Part time or full time employment & $26 \%$ \\
\hline
\end{tabular}

other), the 2 most common concerns raised in these focus groups were the disruption in daily activities by, and time spent on, receiving daily injections of Filgrastim (up to 14 daily injections per cycle). This SOQ represents the first systematic and quantitative survey of the burden of frequent injections of hematopoietic growth factor on patients receiving chemotherapy. The SOQ has not been validated, and formal studies are underway to assess its validity and reliability and to examine the relationship between the SOQ and several cancer-specific healthrelated quality of life instruments.

The extent of disruption of daily activities was measured in terms of time spent travelling to and from the clinic. Patient concerns and attitudes toward frequent injections were assessed on a self-rating scale of 1 to 5 , with 1 representing the least amount of disruption, interference or time commitment, and 5 the greatest amount of disruption, interference or time commitment. Patients also rated the extent of physical discomfort associated with injection on a self-rating scale of 0 to 10 , with 0 representing no discomfort and 10 representing extreme discomfort. Also assessed were patient satisfaction with Filgrastim treatment (based on a 1 to 5 scale, with 1 corresponding to not at all satisfied and 5 corresponding to very much satisfied) and preference for a longer-acting drug. Selected patient responses were grouped into various categories for presentation of data. The use of various scales was intended to provide insight into the degree of effect of treatment on patients' lives. However, responses were neither weighted nor compared with each other, and a total score was not calculated. Participating study sites were the Cancer Center of Albany Medical Center, Northern Indiana Cancer Research Consortium (NICRC), and University of California Los Angeles (UCLA) in the United States.

\section{Statistical methods}

Analysis was performed only on completed questionnaires; no imputation of data was made. Descriptive statistics were performed for each question on the SOQ. Items were analyzed by site and chemotherapy cycle (first or second). Results from both chemotherapy cycles and from all 3 sites were combined for the analyses. Means and standard deviations (SD) were calculated for continuous responses, and frequencies were calculated for categorical responses. The statistical association between the demographic characteristics listed in Table 1 and patient responses listed in Table 2 was calculated using the chisquare test.

\section{Results}

Thirty patients completed the first administration of the SOQ across all 3 sites (6 patients at Cancer Center of Albany Medical Center; 7 at NICRC; and 17 at UCLA). Twenty-four patients completed the second administration of the SOQ. Demographic characteristics were similar among the study centers and are provided in Table 1 . The average age of participants was approximately 61 years (range, 21 to 80 years) and $70 \%$ were female, reflecting the fact that most of the patients at the study sites were older women with breast cancer. Nearly half of the patients reported college level education or above; $45 \%$ of participants had Medicare insurance and/or annual household income greater than $\$ 50,000$. Approximately one quarter of the enrolled participants were employed in some capacity.

Patients received injections ranging in frequency from 1 to 15 per chemotherapy cycle. The effect of daily Filgrastim injections on quality-of-life issues is shown in Table 2. The mean (SD) time commitment for daily Filgrastim treatment was 78 (72) minutes, including 60 (48) minutes on travel to and from the clinic and 18 (24) minutes waiting at the clinic per visit. Most patients (60\%) drove themselves to the clinic for their injection, while $40 \%$ were accompanied by a friend or relative. Forty-five percent of patients were moderately to extremely bothered by travel during chemotherapy cycle 1 ( $\geq 3$ on the 5-point scale); this percentage increased to $59 \%$ during the subsequent cycle.

Forty-four percent of patients reported that the frequency of Filgrastim injections interfered with their daily activities at least some to all of the time ( $\geq 3$ on the 5-point 
Table 2: Summary of selected patient responses to the SOQ

\begin{tabular}{ll}
\hline Effect of treatment on time commitment & $60(48)$ minutes per visit \\
$\begin{array}{l}\text { Mean (SD) travel time to/from the clinic } \\
\text { Mean (SD) waiting time at the clinic }\end{array}$ & $18(24)$ minutes per visit \\
$\begin{array}{l}\text { Effect of treatment on patient attitude } \\
\text { Attitude towards travel for treatment during first chemotherapy cycle }\end{array}$ & $\begin{array}{l}\text { Moderately to extremely bothered, } 45 \% \\
\text { Attitude towards travel for treatment during second chemotherapy cycle }\end{array}$ \\
$\begin{array}{l}\text { Disruption of daily activities caused by injection frequency } \\
\text { Reported to extremely bothered, } 59 \%\end{array}$ \\
$\begin{array}{l}\text { Disruption of work schedule caused by injection frequency } \\
\text { Effect of treatment on patient satisfaction and drug preference time, } 44 \%\end{array}$ \\
$\begin{array}{l}\text { Experience with Filgrastim therapy after most recent chemotherapy cycle } \\
\text { Choice between daily injections versus newer, longer-acting drug }\end{array}$ & $\begin{array}{l}\text { Moderately to very much satisfied, }>90 \% \\
\text { Preference for new drug, } 89 \%\end{array}$
\end{tabular}

scale), and the same percentage indicated they had to rearrange their schedules for medical visits at least some to all of the time. Eighteen percent of working patients took time off from work for injections at least some to all of the time.

The associations between demographic characteristics (Table 1) and selected patient responses to the SOQ (Table 2) were analyzed retrospectively. Younger patients $(<65)$, compared with older $(\geq 65)$ patients, were more likely to take time off from work (31\% vs $4 \%$, respectively; [p $<0.01])$ for Filgrastim administration. In addition, although statistical significance was not achieved, a similar trend was observed wherein younger patients were more likely than older patients to miss appointments (18.5\% vs $8 \%$, respectively; [p $=0.13]$ ) for Filgrastim administration. Finally, a higher percentage of younger patients, compared with older patients, reported that they would have used the time that was spent travelling to and from the clinic to work for pay (36\% vs $16 \%$, respectively; [p $<0.05])$. Apart from age, no significant associations were found between the other demographic characteristics (eg, gender, level of education, insurance coverage, annual household income, employment) and selected patient responses to the SOQ.

Physical discomfort was the least burdensome to patients when compared with the other variables measured. The majority of patients reported little to no discomfort due to injections. The mean (SD) physical discomfort score associated with the injection was 2.1 (2.5) on a 0 to 10 scale.

Over $90 \%$ of the patients were "moderately" to "very much" satisfied with Filgrastim therapy. However, $89 \%$ of the patients who had received daily Filgrastim injections reported that they would prefer a longer-acting drug that required fewer injections.

\section{Discussion}

This study was conducted in oncology patients receiving chemotherapy and Filgrastim. The study assessed the impact of frequent Filgrastim injections on the quality of life of patients receiving chemotherapy and, in particular, the challenges associated with frequent clinic visits.

Results from this study indicate that the majority (89\%) of patients who had received daily Filgrastim injections would prefer a longer-acting therapy that required fewer injections, and thus fewer trips to the clinic. A wide variety of burdens on patient quality of life due to injections were identified in the study. In particular, the frequency of appointments and travelling to and from the clinic were the 2 most common disruptions, as reported by greater than $70 \%$ of the patients.

As patients' lives are disrupted by cancer therapy, treatments that allow a more normal daily routine could improve their quality of life. Patients, as well as their caregivers, could benefit from treatments that achieve optimal outcomes with the least amount of intrusion into their lives. Pegfilgrastim has recently been approved by the FDA for the prevention of neutropenia induced by chemotherapy, and has been shown in clinical studies to be comparable to Filgrastim in efficacy and safety. However, unlike Filgrastim, which requires daily injections when prescribed as needed, longer-acting pegfilgrastim is administered only once per chemotherapy cycle. In our study, patients reported an average time commitment of 1.3 hours per injection visit. Furthermore, two fifths of the patients were accompanied to the clinic by a relative or friend; however, the time spent by a caregiver per injection visit was not determined. Thus, the total time commitment would likely be greater if caregiver time per visit had also been reported. The possibility of saving time on injections in order to attend to other activities of daily life would be of obvious appeal not only to patients, but to caregivers as well. 
The results from this study with Filgrastim can be extrapolated to other medications that also require frequent administration. Darbepoetin alfa, a recently approved erythropoietic agent, can be administered less frequently than Epoetin alfa due to its approximately 3-fold longer half-life. Currently, patients receiving chemotherapy require 1 to 3 injections of Epoetin alfa a week [5], while in clinical studies, darbepoetin alfa was effective when injected as infrequently as every 2,3 , or 4 weeks [8-12]. Both pegfilgrastim and darbepoetin alfa represent a treatment advance in supportive care in oncology by providing patients the benefits of less frequent injections.

This study may have underestimated the real burden of frequent injections to the broader cancer patient population, since the study participants were, on average, older (61 years) and only a quarter of them were employed. A younger patient population may have experienced considerably more inconvenience caused by frequent trips to the clinic since a greater percentage of these patients would be potentially working, caring for children, and involved in a great number of personal and family activities that require their participation. As was observed in this study, younger patients were more likely than older patients to take time off from work for Filgrastim treatment. Other demographic factors may be associated with patient responses; however, they were not found in this study, possibly due to the small sample size. Another potential limitation of the study is that the time spent by caregivers in assisting patients was not collected, which could also contribute to an underestimation of the burden of frequent injections.

Finally, it has been suggested previously that the need to make multiple visits increases the risk of missing required injections, as reported in the setting of administering vaccinations [13]. The effect of frequency of oral medications on treatment compliance has been demonstrated for antihypertensive medications with patients showing higher compliance with simpler regimens [14]. The potential impact of fewer injections on a patient's ability to comply with complete Filgrastim therapy and other treatment for their disease would be of further interest.

\section{Conclusions}

Although the SOQ remains to be validated, the results from this study indicate that travelling to the clinic to receive frequent injections of supportive care medications is a burden to patients with cancer and negatively affects their quality of life. Although current treatments are clinically adequate, new long-acting therapeutics have the potential to improve significantly the quality of patients' lives by requiring fewer injections. Patients should be made aware of these newer alternatives which may help to decrease the burden of treatment in their lives. A controlled trial comparing these newer agents with current treat- ments would help to more accurately identify the impact of frequent injections in patients receiving chemotherapy.

\section{Competing interests}

None declared by SH, CR, and HL

JL is an employee of Amgen, Inc. GHL received grant and other financial support from Amgen and Ortho Biotech.

\section{Authors' contributions}

SH and CR assisted in the design of the questionnaire. JL coordinated the study. HL and JL analyzed the data. GHL assisted in the conduct of the study and contributed to the manuscript. All authors read and approved the final manuscript.

\section{Additional material}

\section{Additional File 1}

Subject Outcome Questionnaire. The questionnaire used in the study is provided in this Adobe Acrobat file.

Click here for file

[http://www.biomedcentral.com/content/supplementary/14726955-2-2-S1.pdf]

\section{References}

I. Crawford J, Ozer H, Stoller R, Johnson D, Lyman G, Tabbara I, Kris M, Grous J, Picozzi V, Rausch G, Smith R, Gradishar W, Yahanda A, Vincent $M$, Stewart $M$ and Glaspy J: Reduction by granulocyte colony-stimulating factor of fever and neutropenia induced by chemotherapy in patients with small-cell lung cancer. $N$ Engl J Med 1991, 18:164-170.

2. Trillet-Lenoir V, Green J, Manegold C, Von Pawel J, Gatzemeier U, Lebeau B, Depierre A, Johnson P, Decoster G, Tomita D and Ewen $C$ : Recombinant granulocyte colony stimulating factor reduces the infectious complications of cytotoxic chemotherapy. Eur J Cancer 1993, 29A:319-324.

3. Rizzo JD, Lichtin AE, Woolf SH, Seidenfeld J, Bennett CL, Cella D, Djulbegovic B, Goode MJ, Jakubowski AA, Lee SJ, Miller CB, Rarick $M U$, Regan DH, Browman GP and Gordon MS: Use of epoetin in patients with cancer: evidence-based clinical practice guidelines of the American Society of Clinical Oncology and the American Society of Hematology. Blood 2002, 100:2303-2320.

4. Demetri GD, Kris M, Wade J, Degos L, Cella D and for the Procrit Study Group: Quality-of-life benefit in chemotherapy patients treated with epoetin alfa is independent of disease response or tumor type: results from a prospective community oncology study. J Clin Oncol 1998, 16:34I2-3425.

5. Gabrilove JL, Cleeland CS, Livingston RB, Sarokhan B, Winer E and Einhorn LH: Clinical evaluation of once-weekly dosing of epoetin alfa in chemotherapy patients: improvements in hemoglobin and quality of life are similar to three-times-weekly dosing. J Clin Oncol 200I, 19:2875-2882.

6. Green MD, Koelbl H, Baselga J, Galid A, Guillem V, Gascon P, Siena S, Lalisang RI, Samonigg H, Clemens MR, Zani V, Liang BC, Renwick J and Piccart MJ: A randomized double-blind multicenter phase III study of fixed-dose single-administration pegfilgrastim versus daily filgrastim in patients receiving myelosuppressive chemotherapy. Ann Oncol 2003, I 4:29-35.

7. Holmes FA, O'Shaughnessy JA, Vukelja S, Jones SE, Shogan J, Savin M, Glaspy J, Moore M, Meza L, Wiznitzer I, Neumann TA, Hill LR and Liang BC: Blinded, randomized, multicenter study to evaluate single administration pegfilgrastim once per cycle versus daily filgrastim as an adjunct to chemotherapy in patients 
with high-risk stage II or stage III/IV breast cancer. J Clin Oncol 2002, 20:727-73।.

8. Thames W, Dozier N, Alley J and Yao B: Initial experience with darbepoetin alfa administered at a fixed dose of $200 \mathrm{mcg}$ every 2 weeks (Q2W) in anemic cancer patients undergoing chemotherapy [abstract]. Blood Proc 2002, 100: 18b.

9. Mirtsching B, Charu V, Vadhan-Raj S, Colowick AB, Rossi G, Tomita $D$ and McGuire WP III: Every-2-week darbepoetin alfa is comparable to rHuEPO in treating chemotherapy-induced anemia. Oncol 2002, 10(suppl):3I-36.

10. Glaspy JA, Jadeja JS, Justice G, Kessler J, Richards D, Schwartzberg L, Tchekmedyian NS, Armstrong S, O'Byrne J, Rossi G and Colowick $A B$ : Darbepoetin alfa given every I or 2 weeks alleviates anaemia associated with cancer chemotherapy. $\mathrm{Br} J$ Cancer 2002, 87:268-276.

I I. Kotasek D, Albertsson M, Mackey J, Steger G, Rossi G, O'Byrne J and Colowick A: Once per cycle dosing of darbepoetin alfa is feasible in anemic cancer patients receiving chemotherapy [abstract]. Ann Oncol 2002, 13:170.

12. Kotasek O, Steger G, Faught W, Underhill C, Poulsen E, Colowick AB, Rossi G, Mackey J and for the Aranesp ${ }^{T M}$ 98029I Study Group: Darbepoetin alfa administered every 3 weeks alleviates anaemlia in patients with solid tumours receiving Chemotherapy; results of a double-blind, placebo-controlled, randomised study. Eur J Cancer 2003, 39:2026-2034.

13. Pellissier JM, Coplan PM, Jackson LA and May JE: The effect of additional shots on the vaccine administration process: results of a time-motion study in 2 settings. Am J Managed Care 2000, 6:1038-1044.

14. Andrejak M, Genes N, Vaur L, Poncelet P, Clerson P and Carre A: Electronic pill-boxes in the evaluation of antihypertensive treatment compliance: comparison of once daily versus twice daily regimen. Am J Hypertens 2000, 13:184- 190.

\section{Pre-publication history}

The pre-publication history for this paper can be accessed here:

http://www.biomedcentral.com/1472-6955/2/2/prepub

Publish with Bio Med Central and every scientist can read your work free of charge

"BioMed Central will be the most significant development for disseminating the results of biomedical research in our lifetime. "

Sir Paul Nurse, Cancer Research UK

Your research papers will be:

- available free of charge to the entire biomedical community

- peer reviewed and published immediately upon acceptance

- cited in PubMed and archived on PubMed Central

- yours - you keep the copyright
BioMedcentral 